\title{
Le financement public des cultes à l'épreuve du pluralisme en Belgique
}

\section{Caroline Sägesser}

\section{(2) OpenEdition}

\section{Journals}

Édition électronique

URL : http://journals.openedition.org/rdr/1042

DOI : $10.4000 /$ rdr. 1042

ISSN : 2534-7462

Éditeur

Presses universitaires de Strasbourg

Édition imprimée

Date de publication : 10 mai 2016

Pagination : 85-99

ISBN : 978-2-86820-954-2

ISSN : 2493-8637

Référence électronique

Caroline Sägesser, "Le financement public des cultes à l'épreuve du pluralisme en Belgique », Revue du droit des religions [En ligne], 1 | 2016, mis en ligne le 12 février 2020, consulté le 19 novembre 2020 URL : http://journals.openedition.org/rdr/1042 ; DOI : https://doi.org/10.4000/rdr.1042

\section{(c) (7) (5)}

La revue du droit des religions est mise à disposition selon les termes de la Creative Commons Attribution - Pas d'Utilisation Commerciale 4.0 International - CC BY-NC 4.0. 


\section{LE FINANCEMENT PUBLIC DES CULTES À L'ÉPREUVE DU PLURALISME EN BELGIQUE}

\section{Caroline SÄGESSER}

Université libre de Bruxelles, Centre interdisciplinaire d'étude des religions et de la läicié (CIERL)

\section{RésuMÉ}

Inchangé dans ses principes depuis 1831, le financement public des cultes en Belgique est un régime d'une grande stabilité dont la seule évolution majeure réside dans l'élargissement des bénéficiaires. Dans un contexte marqué par la désaffection vis-à-vis des cultes historiquement implantés et par le maintien d'une certaine méfiance au sein de la population vis-à-vis des religions nouvelles, cet élargissement alimente un débat relatif tant aux modalités de l'organisation du financement public que de la légitimité de son principe. Cependant, à l'exception du transfert de certaines compétences aux Régions, les réformes acquises ou considérées sont peu nombreuses.

\section{ABSTRACT}

State funding of religions hasn't undergone any major change since 1831, except for the extension of its beneficiaries. In a context of deep secularization and disaffection towards historically established religions groups, coupled with distrust among the population towards newer religious, this enlargement fuels a debate concerning both the way state funding is administered and the legitimacy of the principle itself. However, except for the transfer of certain competencies to the Regions, attempts to introduce significant reforms have proved unsuccessful. 
T e financement public des cultes trouve sa source dans la législation Lnapoléonienne que le régime hollandais (1815-1830) avait laissé subsister. Dans la Belgique indépendante, la loi du 18 germinal an X et le décret impérial du 30 décembre 1809 sur les fabriques d'église continuèrent à être observés, bien que la Constitution de février 1831 ait aboli le Concordat, contraire à la séparation de l'Église et de l'État établie par l'article $16^{1}$. La Constitution belge a inscrit le principe du financement public des cultes en son article 117 (aujourd'hui 181) : « Les traitements et pensions des ministres des cultes sont à la charge de l'État; les sommes nécessaires pour y faire face sont annuellement portées au budget $»^{2}$.

Durant la seconde moitié du XIX siècle, le financement public des cultes, et en particulier de l'Église catholique, a été un objet de discorde entre le parti catholique et le parti libéral (rejoint par le parti socialiste après 1885) ${ }^{3}$. Parmi les griefs formulés à l'encontre du système figurait le déficit de contrôle sur la gestion des fabriques d'église. Une solution de compromis fut adoptée avec la loi du 4 mars 1870 sur le temporel des cultes ${ }^{4}$, qui établit une procédure de contrôle des budgets et des comptes pour les fabriques d'églises et pour les établissements assimilés des cultes protestant, anglican et israélite. Depuis, cette loi constitue la base juridique de l'organisation du financement des cultes autres que catholiques: c'est par une inscription dans la loi du 4 mars 1870 que les cultes islamique et orthodoxe seront ultérieurement « reconnus ». Les obligations des communes et des provinces à l'égard des fabriques d'église et des établissements assimilés des autres cultes dits reconnus ont été, dès 1836, inscrites dans les lois communale et provinciale.

Les organisations convictionnelles reconnues bénéficient également d'autres formes de financement public, notamment pour l'assistance spirituelle

1. Aujourd'hui art. 21: «L'État n'a le droit d'intervenir ni dans la nomination ni dans l'installation des ministres d'un culte quelconque, ni de défendre à ceux-ci de correspondre avec leurs supérieurs, et de publier leurs actes, sauf, en ce dernier cas, la responsabilité ordinaire en matière de presse et de publication. Le mariage civil devra toujours précéder la bénédiction nuptiale, sauf les exceptions à établir par la loi, s'il y a lieu ».

2. À propos de la genèse des dispositions constitutionnelles concernant les cultes, V. TorFs R., « La Belgique et le Luxembourg », in Basdevant-Gaudemet B. et Messner F. (dir.), Les origines historiques du statut des confessions religieuses dans les pays de l'Union européenne, Paris, PUF, 1999, p. 93-118.

3. Sur les querelles du XIX ${ }^{\mathrm{e}}$ siècle, V. Delville J.-P., « Le financement des cultes en Belgique : approche historique », in Husson J.-F. (dir.), Le financement des cultes et de la laïcité : comparaison internationale et perspectives, Éd. namuroises, 2005, p. 79-90 ; SÄGESSER C., Le financement public des cultes dans la Belgique du XIX ${ }^{e}$ siècle, Univ. libre de Bruxelles, à paraître.

4. Loi du 4 mars 1870 sur le temporel des cultes, Moniteur belge, 9 mars 1870. 
ou morale dans des lieux spécifiques tels que l'armée ou les établissements pénitentiaires, et la réalisation et la diffusion d'émissions concédées par les opérateurs publics de radio et de télévision, en vertu de dispositifs législatifs ou réglementaires spécifiques. Les lieux affectés au culte ou à l'assistance morale laique sont par ailleurs exemptés du paiement du précompte immobilier (taxe foncière $)^{5}$.

\section{L'ÉLARGISSEMENT DES BÉNÉFICIAIRES}

Dès les premières années de l'indépendance belge, le principe du financement public s'est étendu non seulement aux églises protestantes, déjà financées sous le régime précédent, mais également aux cultes anglican et israélite. Progressivement, les modalités de ce financement ont été élargies afin de s'établir sur les mêmes bases que le culte catholique. À cet égard, la loi du 4 mars 1870 a constitué une étape importante en permettant à ces cultes de disposer de la personnalité juridique. À la fin du $x^{e}$ siècle, deux autres cultes ont été inscrits au bénéfice des dispositions de la loi de 1870 : le culte islamique en 1974 et le culte orthodoxe en $1985^{6}$. Dans les deux cas, il s'agissait de reconnaître la présence et l'apport des immigrés en provenance du bassin méditerranéen établis en Belgique après la deuxième guerre mondiale.

Si l'organisation du culte orthodoxe n'a rencontré que peu de difficultés, les différentes Églises acceptant l'autorité du métropolite-archevêque représentant le patriarcat de Constantinople, celle de l'islam a été lente et complexe, en l'absence d'un clergé et de tout organe représentatif. Ce n'est qu'en 2007, plus de 30 ans après la reconnaissance du culte islamique, que les premières mosquées ont été admises au bénéfice du financement public par les Régions. La mise en place d'un organe représentatif du culte islamique a connu de nombreuses vicissitudes, et fut le cadre d'une implication forte des autorités publiques, dont la compatibilité avec le prescrit de l'article 21 de la Constitution

5. À propos de l'organisation du financement, V. Husson J.-F., « Le financement public des cultes, de la laïcité et des cours philosophiques », Courrier hebdomadaire du Crisp, n 1703-1704, 2000 ; SÄGESSER C. et SCHREIBER J.-P. (dir.), Le financement public des religions et de la laïcité en Belgique, Bruxelles, Academia-Bruylant, 2010.

6. Loi du 19 juillet 1974 portant reconnaissance des administrations chargées de la gestion du temporel du culte islamique, Moniteur belge, 23 août 1974; Loi du 17 avril 1985 portant reconnaissance des administrations chargées de la gestion du culte orthodoxe, Moniteur belge, 11 mai 1985. L'ensemble des textes concernant les communautés convictionnelles en Belgique sont réunis dans Christians L. L. et DE PoOter P., Code belge droit et religions, Bruxelles, Bruylant, 2005. Une nouvelle édition est en préparation. 
a été interrogée ${ }^{7}$. Deux processus électoraux ayant impliqué l'ensemble de la communauté musulmane de Belgique (en 1998 et en 2005) ont débouché sur la constitution d'Exécutifs des musulmans de Belgique officiellement reconnus comme interlocuteurs de l'État mais dont les difficultés internes et un déficit de légitimité ont ralenti, voire paralysé, l'action. Début 2014, un nouvel Exécutif a été constitué, par le biais d'une élection interne aux représentants des différentes mosquées présentes sur le territoire belge (reconnues et non reconnues par les pouvoirs publics) ${ }^{8}$. Le nouvel organe exécutif, reconnu par le gouvernement ${ }^{9}$, aura pour tâches principales de mener à bien la reconnaissance de nouvelles mosquées, le développement de la formation des imams et professeurs de religion islamique, et la mise en place d'émissions concédées sur les antennes de la Radiotélévision belge francophone (RTBF). La question de la formation des imams et des professeurs de religion islamique préoccupe les autorités depuis plusieurs années. En Communauté française ${ }^{10}$, suite à un rapport universitaire sur l'état de la question, le ministre de l'Enseignement supérieur, Jean-Claude Marcourt, a installé une commission chargée de faire des propositions en vue de favoriser le développement et la reconnaissance d'un islam moderne en Fédération Wallonie-Bruxelles ${ }^{11}$. La commission est principalement chargée de faire des propositions en matière de formation, le ministre ayant indiqué son désir de voir un cursus universitaire se mettre en place. Son rapport a été remis à la fin de l'année 2015.

Le modèle établi pour une Église catholique structurée en diocèses dirigés par les évêques, interlocuteurs tout désignés de l'État, a été testé également à l'occasion de l'admission au financement public de communautés évangéliques. Le synode de l'Église protestante unie de Belgique (EPUB), héritier d'une

7. Notamment par divers recours auprès du Conseil d'État qui n'ont pas abouti. Sur ce sujet, V. SÄgesser C. et ToRrekens C., « La représentation de l'islam », Courrier hebdomadaire du Crisp, n 1996-1997, 2008.

8. SÄGESSER C., « Un nouvel Exécutif des Musulmans de Belgique », analyse en ligne ORELA, 5 av.2014, http://www.o-re-la.org/ [consulté le 12 févr. 2016].

9. Arrêté royal du 2 avril 2014 portant reconnaissance des membres de l'Exécutif des Musulmans de Belgique, Moniteur belge, 18 avr. 2014.

10. La Belgique est un État fédéral qui comprend des régions et des communautés. Les régions disposent de compétences liées au territoire (ex. développement économique) et les communautés sont compétentes dans les matières liées aux personnes (ex. enseignement). La Communauté française a choisi d'utiliser l'appellation usuelle «Fédération Wallonie-Bruxelles».

11. «Une Commission en Fédération Wallonie-Bruxelles pour un islam moderne de Belgique», communiqué de presse, 18 mars 2015. http://marcourt.wallonie.be/ actualites/ une-commission-en-federation-wallonie-bruxelles-pour-un-islam-modernede-belgique.htm?lng=fr [consulté le 12 févr. 2016]. 
reconnaissance reçue en 1839, a longtemps constitué l'organe représentatif du culte protestant. À la fin du xx siècle, les Églises protestantes évangéliques, non affiliées à l'EPUB, ont réclamé une reconnaissance par les pouvoirs publics, dans un contexte où l'attention portée aux organisations sectaires nuisibles par une commission d'enquête parlementaire leur faisait rechercher une forme de légitimité. Devant l'insistance des pouvoirs publics à disposer d'un seul interlocuteur pour toutes les Églises réformées, et au terme d'un long processus de négociation accompagné, là aussi, par les autorités, un nouvel organe représentatif pour le culte protestant-évangélique a été constitué le

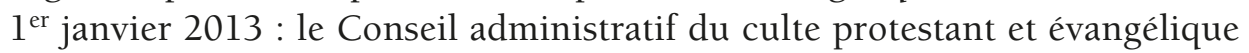
$(\mathrm{CACPE})^{12}$. Depuis la constitution du CACPE, une vingtaine de nouvelles Églises protestantes évangéliques ont été admises au bénéfice du financement public.

L'organisation de l'islam et la restructuration du culte protestant-évangélique ont mis en lumière une double difficulté : tout d'abord, celle d'appliquer à des cultes dépourvus d'une hiérarchie ecclésiale et très diversifiés dans leur composition, que ce soit sur le plan de la théologie, de la langue utilisée ou de l'origine des fidèles, un modèle d'organisation du culte hérité des dispositions concordataires visant l'Église catholique ${ }^{13}$. Ensuite, les difficultés rencontrées par les communautés cultuelles pour constituer leurs organes représentatifs, dits chefs de culte, ont amené les pouvoirs publics à s'impliquer dans ce processus, en contradiction avec le principe constitutionnel de séparation.

L'élargissement à la communauté philosophique non confessionnelle, familièrement dénommée " laïcité organisée », a constitué un changement de plus large portée. En l'absence d'une loi de portée similaire à la loi française de séparation de 1905 et sans inscription de la laïcité parmi les principes constitutionnels, les nombreuses associations laïques qui s'étaient constituées au XIX ${ }^{e}$ siècle pour lutter contre l'influence de l'Église catholique et établir la laiicité de l'État, ont subsisté. Dans un contexte marqué par la sécularisation et la perte par l'Église de son rôle d'acteur sociétal important, les associations laïques ont développé, à côté de leurs revendications politiques, une conception philosophique de la laicité, définie comme une « conception de vie qui se fonde sur l'expérience humaine, à l'exclusion de toute référence confessionnelle, dogmatique ou surnaturelle, qui implique l'adhésion aux valeurs du

12. Statuts du CACPE : http://cacpe.be/cacpe/statuts/ [consulté le 12 févr. 2016].

13. Sur la difficulté d'organiser la représentation des cultes minoritaires, V. OverbeEke A., "Eenheids-vertegenwoordiging van erkende religies in het Belgische eredienstrecht», Recht, Religie en Sameleving, 2013/2, p. 5-43. 
libre examen, d'émancipation à l'égard de toute forme de conditionnement et aux impératifs de citoyenneté et de justice ${ }^{14}$. La laïcité organisée rassemble aujourd'hui des communautés d'athées ou d'agnostiques, fédérées au sein d'un Conseil central laique, dont le Centre d'action laique (CAL) est la branche francophone. Depuis les années 1970, le mouvement laïque a développé une revendication de reconnaissance et d'accès au financement public au même titre que les cultes. Cette revendication a été rencontrée en deux temps. En 1993, une modification de la Constitution a inscrit l'obligation pour l'État de financer les traitements des délégués qui offrent une assistance morale ${ }^{15}$. En 2002, une loi a organisé le financement du Conseil central laiqque, de ses délégués et de ses implantations locales sur une base semblable à celle du financement des cultes: l'État fédéral paie les traitements et les pensions des délégués laiques, et les provinces sont chargées d'éponger le déficit des établissements de l'assistance morale laïque ${ }^{16}$. Cet élargissement du financement public à la communauté philosophique non confessionnelle a suscité au sein du monde laique des oppositions contre cette transformation d'un mouvement de revendication d'un idéal laique de portée universelle en une simple organisation participant à la pluralité des convictions. Il a également contribué à la pérennisation d'un système de financement public qui était ainsi devenu plus respectueux de l'égalité entre les citoyens ${ }^{17}$.

L'augmentation des bénéficiaires du financement public a naturellement un impact sur l'évolution des budgets affectés aux cultes : le budget des provinces est le plus affecté, eu égard à ses obligations à l'égard des mosquées et des établissements d'assistance morale, récemment reconnus. Le budget fédéral évolue plus modestement : la baisse régulière du nombre de ministres du culte catholique, en raison du décès ou du départ à la pension de nombre d'entre eux, qui ne sont pas remplacés, compense partiellement la création

14. Article 4 des statuts du Centre d'action laiqque (CAL), http://laicite.be/le_cal/2_4_2_les_ statuts [consulté le 12 févr. 2016].

15. L'article 181 s'est enrichi d'un deuxième paragraphe : « $\S 2$. Les traitements et pensions des délégués des organisations reconnues par la loi qui offrent une assistance morale selon la conception philosophique non confessionnelle sont à la charge de l'État; les sommes nécessaires pour y faire face sont annuellement portées au budget. »

16. Loi du 21 juin 2002 relative au Conseil central des Communautés philosophiques non confessionnelles de Belgique, aux délégués et aux établissements chargés de la gestion des intérêts matériels et financiers des communautés philosophiques non confessionnelles reconnues, Moniteur belge, 22 oct. 2002.

17. SÄGESSER C. et Husson J.-F., « La reconnaissance et le financement de la laïcité », Courrier hebdomadaire du Crisp, n 1756 et 1760, 2002. 
de nouveaux postes auprès des cultes islamique et protestant-évangélique et l'arrivée des conseillers moraux ${ }^{18}$.

\section{L'IMPACT DE LA TRANSFORMATION EN ÉTAT FÉDÉRAL}

La transformation de la Belgique en État fédéral, acté dans la Constitution en 1994, octroyant progressivement davantage de compétences aux entités fédérées, a emporté la législation sur les fabriques d'église en 2002 : la régionalisation des lois communale et provinciale a confié aux Régions les compétences en matière de fabriques d'église et d'établissements chargés de la gestion du temporel des cultes reconnus ${ }^{19}$.

Désormais, l'État fédéral est compétent pour la reconnaissance d'un culte et pour le paiement des traitements et salaires de ses ministres, ainsi que pour l'ensemble de la législation relative à la laïcité organisée, qui n'a pas fait l'objet de cette régionalisation, tandis que les Régions sont compétentes pour la reconnaissance des communautés locales et leur financement ${ }^{20}$. Ce partage des responsabilités exige une collaboration entre les différents niveaux de pouvoir, qui a été formalisée par la conclusion d'un accord de coopération ${ }^{21}$. Cet accord instaure des procédures de consultation entre l'État fédéral et les Régions, tant pour la reconnaissance d'un nouveau culte au niveau fédéral que pour celle d'une communauté locale au niveau régional. Le financement des communautés locales (fabriques d'église et établissements assimilés pour les autres organisations convictionnelles) est à charge des communes pour

18. Les moyens budgétaires alloués aux cultes et à la laïcité au Service public fédéral de la Justice sont passés de 91,5 millions d'euros en 2001 à 106 millions d'euros en 2014. Source : Husson J.-F., « Les montants affectés aux cultes et à la laïcité », in SCHREIBER J.-P. et SÄGESSER C. (éd.), Le financement public des religions et de la lä̈cité en Belgique, Bruxelles, Academia Bruylant, 2010, p. 53 et Sägesser C., SChreiber J.-P. et VAnderPelen-Diagre C., Les religions et la laïcité en Belgique. Rapport 2013, Bruxelles, ORELA, avril 2014, p. 55.

19. Loi spéciale du 13 juillet 2001 portant transfert de diverses compétences aux régions et communautés, Moniteur belge, 3 août 2001.

20. Sur ce sujet, V. Amez F., «Un aspect oublié de la réforme de l'État: le régime des cultes », Journal des tribunaux, 2002, p. 529-537; Dujardin J. et Vandenbossche E., « De regionalisering van de bestuurinstellingen van de erkende erediesnten », Tijdschrift voor bestuurswetenschappen en publiek recht, 2002, p. 447-453 et SÄGESSER C., « Le temporel des cultes depuis sa régionalisation », Courrier hebdomadaire du Crisp, n 1968, 2007.

21. Accord de coopération entre l'Autorité fédérale, la Région flamande, la Région wallonne et la Région de Bruxelles-Capitale en ce qui concerne la reconnaissance des cultes, les traitements et pensions des ministres des cultes, les fabriques d'église et les établissements chargés de la gestion du temporel des cultes reconnus, Moniteur belge, 14 juin 2004. En juillet 2008, l'accord a été étendu à la Communauté germanophone. 
les fabriques d'église et les administrations des cultes protestant-évangélique, anglican et israélite ; il est à charge des provinces pour les fabriques cathédrales du culte catholique, et les administrations des cultes islamique et orthodoxe ainsi que celles de la laïcité organisée.

La législation a été modifiée dans les trois Régions ${ }^{22}$. La Flandre est la première à avoir adopté un décret en la matière, qui a aboli le décret du 30 décembre 1809 et la loi du 4 mars 1870, tout en conservant les mécanismes contenus dans l'ancienne législation : le décret prévoit la couverture du déficit des fabriques d'église et autres établissements assimilés par les communes ou les provinces. Il innove en prévoyant la création d'une administration centrale au niveau communal pour le culte qui dispose de plus de quatre paroisses (ou structures assimilées) dans la commune, la rédaction d'un plan budgétaire pluriannuel, la réduction du nombre de fabriciens, l'abolition du bureau des marguilliers, l'introduction d'une concertation au moins bisannuelle entre la commune et l'administration centrale ou les conseils de fabrique et assimilés et l'introduction d'une possibilité de suspension, par le collège des bourgmestre et échevins, d'un acte de la fabrique ou de l'établissement assimilé qui porterait préjudice à l'intérêt communal ${ }^{23}$.

L'esprit général du décret est celui d'un renforcement du contrôle, particulièrement au niveau communal, et d'une volonté de rationalisation de la gestion. Le décret ne fait pas mention de l'obligation pour la commune de fournir un logement au desservant. Celle-ci est cependant reprise dans un autre instrument, le décret flamand sur les communes du 15 juillet $2005^{24}$. Le décret sur les provinces prévoit la même obligation à l'égard des cultes organisés sur le plan provincial ${ }^{25}$.

22. À noter que la Région wallonne a transféré l'exercice de cette compétence à la Communauté germanophone, pour la petite région de langue allemande. La Communauté germanophone a adopté ses propres normes législatives et en particulier le décret du 19 mai 2008 relatif à l'organisation matérielle et au fonctionnement des cultes reconnus, Moniteur belge, $1^{\text {er }}$ sept. 2009.

23. Décret flamand du 7 mai 2004 relatif à l'organisation matérielle et au fonctionnement des cultes reconnus, Moniteur belge, 6 sept. 2004.

24. Décret communal du 15 juillet 2005, Moniteur belge, 31 août 2005. L'article 151 prévoit que le budget comprend obligatoirement « les allocations communales aux pouvoirs locaux des cultes reconnus, visés au décret du 7 mai 2004 relatif à l'organisation matérielle et au fonctionnement des cultes reconnus, conformément aux dispositions y afférentes et l'indemnité pour frais de logement des ministres des cultes s'il n'est pas prévu de logement conformément aux dispositions en vigueur en la matière ».

25. Décret provincial du 9 décembre 2005, Moniteur belge, 29 déc. 2005. 
En septembre 2005, un arrêté a déterminé les critères de reconnaissance des nouvelles communautés locales ${ }^{26}$. La communauté doit notamment justifier de sa «pertinence sociale » et de « son engagement dans l'ensemble de la communauté locale au sein du ressort territorial ». Différents engagements écrits sont également exigés de la communauté en matière de respect de la Constitution et de la Convention européenne des droits de l'homme. Le gouvernement flamand a ainsi établi des critères de reconnaissance qui s'appliquent aux communautés locales de tous les cultes reconnus, même si, de facto, ils ont concerné essentiellement les communautés islamiques, et qui reposent largement sur la démonstration de leur utilité sociale et de leur intégration dans la société flamande. L'ancienne justification défendue pour l'Église catholique, à savoir que le financement des fabriques d'église était une juste compensation à la confiscation des biens ecclésiastiques et à la suppression de la dîme, lors de la Révolution française, a disparu.

En juillet 2012, la Région flamande a modifié sa législation, prévoyant notamment une procédure de reconnaissance des fusions de paroisses du culte catholique ${ }^{27}$. Ces nouvelles dispositions s'inscrivent dans le cadre d'un processus de réaffectation des églises, soutenu par un décret qui permet aux autorités locales et aux fabriques d'église de recevoir des subventions pour la réaffectation totale ou partielle des églises qui ne sont plus beaucoup utilisées ${ }^{28}$.

En Wallonie, la réforme de la législation sur le temporel des cultes a tardé. Dans un premier temps, le gouvernement a pris, sur la base de l'ancienne législation, des arrêtés destinés à permettre la reconnaissance des communautés locales du culte islamique ${ }^{29}$. Bien qu'annoncée au début de la législation 2004-2009, et ayant fait l'objet d'auditions au Parlement wallon entre mai 2005 et juin 2006, la réforme de la loi n'a vu le jour qu'au $1^{\text {er }}$

26. Arrêté du gouvernement flamand établissant les critères d'agrément des communautés d'églises et religieuses locales des cultes reconnus, Moniteur belge, 16 déc. 2005.

27. Décret du 6 juillet 2012 modifiant diverses dispositions du décret du 7 mai 2004 relatif à l'organisation matérielle et au fonctionnement des cultes reconnus, Moniteur belge, 16 août 2012.

28. Décret du 12 juillet 2013 portant octroi de subsides pour bâtiments du culte, bâtiments destinés à l'exercice public de l'assistance morale non confessionnelle et crématoriums, Moniteur belge, 13 août 2013.

29. Arrêtés du gouvernement wallon du 13 octobre 2005 portant organisation des comités chargés de la gestion du temporel des communautés islamiques reconnues, fixant le modèle de règlement d'ordre intérieur des comités chargés de la gestion du temporel des communautés islamiques reconnues et fixant les modèles des budgets et comptes à dresser par les comités chargés de la gestion du temporel des communautés islamiques reconnues, Moniteur belge, 27 oct. 2005. 
janvier 2015. Le gouvernement wallon a opté pour une réforme partielle, qui ne concerne que la tutelle, et non l'organisation des établissements publics des cultes. Le "décret Furlan », du nom du ministre wallon des pouvoirs locaux, insère dans le Code wallon de la démocratie locale et de la décentralisation un nouveau chapitre concernant la tutelle administrative sur les établissements chargés de la gestion du temporel des cultes reconnus. Il modifie, tout en les laissant subsister, le décret impérial du 30 décembre 1809 et la loi du 4 mars 1870 sur le temporel des cultes, afin de les mettre en conformité avec les nouvelles dispositions ${ }^{30}$.

Outre une simplification des procédures, les principales modifications introduites par la nouvelle législation sont l'instauration d'une tutelle des communes sur les budgets et les comptes des fabriques, qu'elles devront désormais approuver, là où un simple avis était auparavant demandé, et l'organisation d'une tutelle générale du gouverneur de province sur les autres actes de la fabrique. Les nouvelles dispositions laissent intactes les obligations financières de la commune à l'égard des fabriques et ne modifient pas non plus l'organisation des fabriques, toujours régie par le décret impérial de 1809.

Les évêques des diocèses wallons et plusieurs fabriques ont introduit un recours en annulation partielle du décret auprès de la Cour constitutionnelle, début octobre $2014^{31}$. Le recours se fonde principalement sur l'ingérence des pouvoirs publics dans l'organisation du culte, dénoncée comme déraisonnable et injustifiée, et le non-respect de la spécificité des établissements cultuels, qui se voient appliquer des règles semblables à celles en vigueur pour les centres publics d'action sociale (CPAS). Le recours n'est pas suspensif.

Dans la Région de Bruxelles-Capitale, qui présente un paysage confessionnel particulièrement diversifié, et dont l'organisation présente une particularité institutionnelle en raison de l'absence de l'échelon provincial (pour les cultes organisés sur cette base, à savoir le culte islamique et le culte orthodoxe, règles d'organisation, financement et contrôle sont dispensés par la même autorité : la Région), aucune réforme en profondeur de la législation n'est encore intervenue, en dépit de l'annonce qui en avait été faite au début de la législation 2009-2014. Seuls un toilettage des textes et l'élaboration d'une

30. Décret du 13 mars 2014 modifiant le Code de la démocratie locale et de la décentralisation et diverses dispositions relatives à la tutelle sur les établissements chargés de la gestion du temporel des cultes reconnus, Moniteur belge, 4 avril 2014.

31. SÄGESSER C., « Nouvelles dispositions à propos des fabriques d'église en Wallonie: les évêques introduisent un recours ", Analyse ORELA en ligne, 14 déc. 2014, http://www.ore-la.org [consulté le 12 févr. 2016]. 
ordonnance permettant la reconnaissance des communautés islamiques locales ont été effectués ${ }^{32}$.

\section{LES RÉFORMES ÉVOQUÉES}

Au niveau fédéral, le gouvernement a entamé en 2005 un processus de réflexion. Une commission chargée d'examiner le statut des ministres des cultes reconnus et de formuler des propositions de réforme a dressé un inventaire des inégalités rencontrées dans le régime des cultes et des organisations philosophiques non confessionnelles, et formulé différentes propositions de réforme, allant jusqu'à envisager le passage à un financement par enveloppes budgétaires, sur la base de critères objectifs qui restaient à déterminer ${ }^{33}$.

Pour dégager une piste de réforme plus concrète, un groupe de travail a ensuite été constitué. Son rapport, déposé en octobre 2010, comprenait un avant-projet de loi dessinant une réforme importante de l'ensemble de la législation sur le temporel des cultes de compétence fédérale ${ }^{34}$. La proposition prévoyait un système d'enregistrement puis de reconnaissance des organisations convictionnelles sur base d'une série de critères. Pour répartir les budgets

32. Lordonnance du 18 juillet 2002 portant diverses réformes en vertu de la loi spéciale du 13 juillet 2001 portant transfert de diverses compétences aux régions et aux communautés (Moniteur belge, 7 août 2002) a substitué le gouvernement régional aux anciennes autorités dans les textes existants et en a établi la version néerlandaise; celle du 19 février 2004 portant modification du décret impérial du 30 décembre 1809 concernant les fabriques d'église (Moniteur belge, 18 mars 2004), répondant à " une nécessité urgente » a réduit le nombre de fabriciens de neuf à cinq, supprimé la notion de "notables » catholiques », et supprimé le bureau des marguilliers. Lordonnance du 29 juin 2006 portant diverses dispositions relatives aux cultes reconnus (Moniteur belge, 10 juill. 2006), précise certains points de la législation en vigueur: elle exige la production d'un certificat de bonne vie et mœurs pour les membres des conseils de fabrique et assimilés, établit à 18 ans l'âge minimum pour être élu ou être électeur des dits conseils, soumet la construction ou la modification des édifices à l'approbation du gouvernement ou de la commune, moyennant avis de la commune et présentation d'un plan financier et budgétaire, et affirme que seuls les ministres des cultes rémunérés par l'Autorité fédérale ont droit à la mise à disposition du logement ou au versement de l'indemnité. L'ordonnance du 29 juin 2006 relative à l'organisation et au fonctionnement du culte islamique (Moniteur belge, 10 juill. 2006) instaure une procédure de reconnaissance des communautés islamiques par le gouvernement de la Région de Bruxelles-Capitale, sur proposition de l'organe représentatif du culte islamique reconnu par les autorités fédérales, et sur base d'un dossier dont le gouvernement détermine le contenu.

33. Le financement par l'État fédéral des ministres des cultes et des délégués du Conseil central laïque, rapport de la Commission des sages, 2005-2006.

34. La réforme de la législation surles cultes et les organisations philosophiquesnon-confessionnelles, rapport du Groupe de travail instauré par l'arrêté royal du 13 mai 2009, oct. 2010. 
entre les différentes organisations convictionnelles, le groupe de travail proposait l'organisation périodique d'une vaste enquête scientifique dirigée par un comité de pilotage pluraliste, destinée à photographier le paysage des convictions et des pratiques de la population. La commission de la Justice de la Chambre a examiné le rapport du groupe de travail en février et en mars 2011, dans un double contexte de crise : crise politique, le gouvernement Leterme II étant en affaires courantes depuis près d'un an, et crise au sein de l'Église catholique, marquée par le scandale de la révélation de cas de pédophilie au sein du clergé. Ce scandale, qui a débouché sur la constitution d'une commission spéciale parlementaire pour traiter de ce sujet, a relancé le débat politique, à la fois sur l'opportunité du maintien du financement public des organisations convictionnelles, et sur la part dévolue à l'Église catholique. Dans la foulée, plusieurs propositions de loi émanant de parlementaires libéraux, écologistes et socialistes néerlandophones ont été déposées au Sénat et à la Chambre, pour substituer au système actuel une forme d'impôt philosophiquement dédié ou de répartition des subsides sur la base des résultats d'une enquête. Consulté, le Conseil d'État a estimé difficile de concilier un mécanisme d'impôt dédié avec l'article 181 de la Constitution ${ }^{35}$. Ni la proposition du groupe de travail ni les initiatives parlementaires n'ont débouché sur une réforme. La question paraît avoir quitté l'agenda politique, ainsi qu'en témoigne l'absence de propositions à ce sujet dans les programmes des différentes formations politiques pour les élections législatives du 25 mai 2014.

\section{VERS UNE RÉFORME DE L'ENSEIGNEMENT DE LA RELIGION ET DE LA MORALE?}

Si l'enseignement de la religion et de la morale dans l'enseignement obligatoire est encadré par des dispositifs juridiques distincts, il doit cependant être considéré comme partie intégrante du financement des cultes : les cours de religion, tant dans les écoles dites libres, c'est-à-dire des écoles privées subventionnées, essentiellement catholiques, que dans les écoles officielles, c'est-à-dire organisées par les pouvoirs publics, sont placés sous l'autorité des organes représentatifs des cultes reconnus par l'État, tout en étant intégralement financés par les pouvoirs publics. La place de la religion à l'école a fait l'objet d'âpres conflits entre catholiques et partisans de la laïcisation de

35. Avis 49.285/AG du 26 avril 2011 sur la proposition de loi visant à fixer un critère objectif de répartition du budget finançant les cultes et la laïcité. 
l'enseignement jusque dans les années 1950. En 1958, un Pacte scolaire conclu entre les principales formations politiques a pacifié le débat. Coulées dans une loi l'année suivante ${ }^{36}$, les principales dispositions du Pacte ont augmenté le subventionnement des écoles dites libres, qui sont surtout des écoles liées à l’Église catholique, mis fin à l'influence du clergé dans les écoles communales, et contraint les écoles du réseau officiel à offrir aux parents le choix entre un cours de religion (catholique, protestante ou israélite) et un cours de morale non confessionnelle. Dans les établissements libres subventionnés se réclamant d'un caractère confessionnel, seule la religion correspondant au caractère de l'enseignement demeurait enseignée.

Des cours de religion islamique et orthodoxe ont ensuite été introduits dans les écoles du réseau officiel après la reconnaissance de ces cultes, entre 1976 et 1989. En 1988, la quasi-totalité des compétences en matière d'enseignement ont été transférées aux Communautés. Afin de garantir la paix scolaire et de pérenniser les principales dispositions du Pacte scolaire, ces dernières ont été insérées dans la Constitution. À cette occasion, le concept de "religions reconnues » a fait son entrée dans la Constitution - et dans l'ordre juridique belge tout entier, dont il était jusque-là absent : le quatrième alinéa du premier paragraphe de l'article 24 énonce en effet que «Les écoles organisées par les pouvoirs publics offrent, jusqu'à la fin de l'obligation scolaire, le choix entre l'enseignement d'une des religions reconnues et celui de la morale non confessionnelle ». Cette nouvelle rédaction implique ainsi l'élargissement automatique de l'offre de cours de religion - organisés par les Communautés - à toute nouvelle religion reconnue - par l'État fédéral. Le processus de reconnaissance du bouddhisme, actuellement en cours, pourrait ainsi déboucher, à court ou moyen terme, sur l'organisation de cours de bouddhisme dans les écoles officielles. Il convient cependant de noter que le bouddhisme ayant demandé sa reconnaissance comme philosophie non confessionnelle (deuxième alinéa de l'article 181 de la Constitution), et non comme religion (premier alinéa de l'article 181), on pourrait émettre un doute sur le bénéfice automatique de l'article 24 de la Constitution qui prévoit l'organisation des cours « des religions reconnues et de la morale non confessionnelle $»$ : il serait cependant conforme à l'esprit de l'article 24 de la Constitution d'organiser des cours de bouddhisme.

L'idée qui sous-tendait le Pacte scolaire était que l'éducation reçue à l'école devait, en matière de religion et de morale, constituer le prolongement de celle

36. Loi du 29 mai 1959 modifiant certaines dispositions de la législation de l'enseignement, Moniteur belge, 19 juin 1959. 
reçue à la maison. Le développement du pluralisme confessionnel a rendu le maintien de cette logique difficile. L'augmentation du nombre de religions reconnues a conduit à la diversification de l'offre, qui complique l'organisation pratique de ces cours, notamment au niveau de la fixation des horaires par les directeurs d'établissement. Placés sous l'autorité des organes représentatifs des différents cultes, en vertu de la séparation de l'Église et de l'État établie par l'article 21 de la Constitution, les cours des différentes religions présentent peu de garanties en termes de contenu de programmes, de formation des professeurs et de qualité de l'inspection. En particulier, la qualité de la formation des professeurs de religion islamique est régulièrement questionnée, alors que ce cours est suivi par près d'un élève sur deux dans les écoles officielles de Bruxelles. Le cours de morale non confessionnelle, initialement conçu comme un cours neutre, est placé sous l'autorité du ministère de l'Éducation comme les autres enseignements. Son caractère neutre a cependant été remis en cause par des décisions de justice: le Conseil d'État a estimé dès 1985 que le cours de morale organisé en Communauté flamande n'avait pas ce caractère. Depuis, la possibilité d'une dispense de tout cours dit philosophique existe en Flandre $^{37}$. En Communauté française, un arrêt de la Cour constitutionnelle du 12 mars 2015, répondant à une question préjudicielle adressée par le Conseil d'État, a estimé que l'obligation faite aux parents (ou aux enfants majeurs) de choisir entre l'enseignement d'une des religions reconnues ou de la morale non confessionnelle violait leurs droits fondamentaux. Désormais, les uns et les autres ont le droit, en Communauté française comme en Communauté flamande, d'obtenir une dispense de tout cours de religion ou de morale, qui, selon l'arrêt de la Cour constitutionnelle, ne doit pas être motivée, afin précisément de garantir le respect de la vie privée que l'obligation du choix mettait à $\mathrm{mal}^{38}$. Cette absence de justification, ainsi que la forte médiatisation donnée à l'arrêt de la Cour constitutionnelle, laissent supposer que les demandes de dispenses seront beaucoup plus nombreuses dans les écoles francophones qu'elles ne le sont jusqu'à présent dans les écoles flamandes, où la dispense est une possibilité peu connue et doit toujours être justifiée par l'appartenance à une communauté convictionnelle non représentée dans l'offre de cours proposée. Une circulaire ministérielle a prévu l'organisation de cette dispense dès la rentrée de septembre 2015 et des modifications de la législation sont annoncées.

37. Conseil d'État, arrêt n²5.326 du 14 mai 1985, affaire Sluijs, et arrêt n³5.442 du 10 juill. 1990, affaire Vermeersch.

38. Cour Constitutionnelle, arrêt $n^{\circ}$ 34/2015 du 12 mars 2015, http://www.const-court.be/ public/f/2015/2015-034f.pdf [consulté le 12 févr. 2016]. 
Prenant acte des difficultés organisationnelles du système, constatant les progrès de la sécularisation et la diversification convictionnelle induite par l'immigration ou déplorant l'absence d'un enseignement de la philosophie, diverses propositions de réforme du système ont vu le jour depuis une vingtaine d'années, sans jamais aboutir ${ }^{39}$. Le gouvernement de la Communauté française constitué en juillet 2014 a prévu le remplacement d'une des deux heures hebdomadaires de religion ou de morale dans l'enseignement officiel par un cours de citoyenneté, dès septembre 2016 ${ }^{40}$. Depuis, l'actualité dramatique du début de l'année 2015 a relancé le débat autour de l'opportunité de développer ce cours sur plus d'une heure par semaine, et d'y inclure un enseignement du fait religieux et convictionnel dans toute sa diversité, ayant notamment pour objectif de lutter contre la radicalisation d'origine religieuse.

Tant en matière d'enseignement de la religion et de la morale que d'organisation du temporel des cultes, la Belgique connaît actuellement des modifications à la marge, tantôt pour adapter les instruments législatifs au nouveau paysage institutionnel du pays, tantôt pour s'efforcer d'accompagner les mutations du paysage convictionnel. Le régime belge des cultes (et des organisations philosophiques non confessionnelles) est un élément constitutif de l'organisation du pluralisme dans un pays traversé par de nombreuses tensions. Partie intégrante du compromis qui présida à la naissance de la Belgique en 1831, le financement public des cultes paraît difficile à réformer radicalement dans un pays dont la survie même est parfois remise en cause.

39. V. Sägesser C., "Les cours de religion et de morale dans l'enseignement obligatoire », Courrier hebdomadaire du Crisp, $n^{\circ}$ 2140-2141, 2012.

40. Déclaration de politique communautaire 2014-2019 : «Fédérer pour réussir », 23 juill. 2014, p. 10. 